\title{
ON SMOOTHNESS OF TUKEY DEPTH CONTOURS
}

\author{
IRÈNE GIJBELS ${ }^{1}$ AND STANISLAV NAGY ${ }^{1,2}$
}

\begin{abstract}
The smoothness of Tukey depth contours is a regularity condition often encountered in asymptotic theory, among others. This condition ensures that the Tukey depth fully characterizes the underlying multivariate probability distribution. In this paper we demonstrate that this regularity condition is rarely satisfied. It is shown that even well-behaved probability distributions with symmetrical, smooth and (strictly) quasiconcave densities may have non-smooth Tukey depth contours, and that the smoothness behaviour of depth contours is fairly unpredictable.
\end{abstract}

Key Words and Phrases: data depth, depth contours, optimal halfspace, Tukey depth, smoothness, quasi-concavity.

\section{INTRODUCTION}

In the past years, depth functions for multivariate data proved to be of importance in non-parametric statistics (see, for example, Zuo and Serfling, 2000). In the present paper we address smoothness properties of one of the most important depth functions in the literature, the Tukey (or halfspace) depth (Tukey, 1975).

The original motivation for this research comes from the problem of distribution-bydepth characterization - that is, if there exists a one-to-one mapping between the Tukey depth functions and probability distributions on a Euclidean space. An affirmative answer to this question would justify the usage of depth by itself, and give an impulse to investigate further into depth-based calculus of multivariate distributions. Though the problem is still open in general, a number of promising positive results concerning subclasses of probability distributions have been proved. We mention the work of Struyf and Rousseeuw (1999), Koshevoy (2002), Hassairi and Regaieg (2008), Cuesta-Albertos and Nieto-Reyes (2008) and a recent article by Kong and Zuo (2010). In the latter the authors showed that a probability distribution is characterized by its depth if all the depth contours are smooth.

The condition of smooth Tukey depth contours may appear to be fairly weak. In fact, the opposite turns out to be true. Apart from elliptically symmetrical distributions Kong and Zuo (2010) did not identify any other class of distributions for which the smoothness condition holds. Moreover, Rousseeuw and Ruts (1999) noticed that for a bivariate Cauchy distribution, which has a smooth and strictly unimodal density, there exist infinitely many points at which depth exhibits non-smoothness.

A different motivation for the study of the smoothness of the depth contours arises from the theoretical properties of the Tukey depth. As noticed by various authors, the

\footnotetext{
1 KU Leuven, Department of Mathematics and Leuven Statistics Research Centre (LStAT), Belgium

2 Charles University in Prague, Department of Probability and Math. Statistics, Czech Republic

E-mail addresses: irene.gijbels@wis.kuleuven.be.

Date: January 6, 2017.
} 
asymptotic behaviour of many concepts related to the Tukey depth is driven by the property of the uniqueness of halfspaces realizing the depth. For instance, by a result of Massé (2004, Corollary 2.2), the Tukey depth is asymptotically Gaussian at $\mathbf{x} \in \mathbb{R}^{d}$ if and only if there exists a unique halfspace realizing the depth at $\mathbf{x}$. Such a halfspace is commonly called the optimal halfspace of $\mathbf{x}$ (Romanazzi, 2001). In Proposition 1 in this paper, we show that this condition is in fact equivalent with the smoothness of depth contours at $\mathbf{x}$. Thus, the asymptotic distribution of an empirical depth process is Gaussian if and only if all the depth contours are smooth.

Similarly, the regularity in

- the asymptotic theory for multivariate trimming (Nolan, 1992, p. 162), closely related to the Tukey depth,

- the derivation of the influence function for the Tukey depth (Romanazzi, 2001, 2002), or

- the central limit theorem for the Tukey depth regions (Massé and Theodorescu, 1994, Theorem 4.7)

are all closely tied to the notion of unique optimal halfspaces. This offers another view on how to perceive the points with smooth depth contours - as the points of regularity of empirical depth-related processes. Hence, we shall also refer to points of depth nonsmoothness as to the points of depth irregularity of a probability distribution.

In this paper we address the question whether there are some natural classes of wellbehaved distributions (broader than elliptically symmetrical distributions) such that for each probability distribution $P$ in this class

$$
\text { the Tukey depth contours of } P \text { are smooth at every point } \mathbf{x} \in \mathbb{R}^{d} \text {. }
$$

To this end, we investigate mixtures of Gaussian distributions, $L^{p}$ symmetrical distributions (in line with the research of Dutta et al. (2011)), and finally the class of (strictly) quasi-concave distributions with smooth densities.

The latter class is of special interest, as the Tukey depth itself is always a quasiconcave function (Donoho and Gasko, 1992, Lemma 2.2). This raises the question whether the smoothness of a quasi-concave density is enough for the Tukey depth to copy the behaviour of the underlying density, and reveal a degree of smoothness as well.

In Section 2, a characterization of the points of depth regularity is made, which is used in the sequel for the construction of examples. An example of a mixture of two bivariate Gaussian distributions not satisfying (S) is given in Section 3. In Section 4 it is shown that not even the $L^{p}$ symmetry of a distribution (unless $p=2$, in which case we are dealing with elliptically symmetrical distributions) provides smoothness of depth contours. Finally, in Section 5 we provide examples of (strictly) quasi-concave symmetrical distributions whose depth contours are not smooth either. Concluding remarks can be found in Section 6 .

\section{Smoothness of Depth Contours}

Let $P$ be a continuous probability distribution on $\mathbb{R}^{d}$ with density function $f$. The density $f$ is called quasi-concave, if for all $\mathbf{x}, \mathbf{y} \in \mathbb{R}^{d}, \mathbf{x} \neq \mathbf{y}$ and $\lambda \in(0,1)$

$$
f(\lambda \mathbf{x}+(1-\lambda) \mathbf{y}) \geq \min \{f(\mathbf{x}), f(\mathbf{y})\} .
$$

If the inequality in (1) holds strictly, then we say that $f$ is strictly quasi-concave. The geometrical interpretation of quasi-concavity is that the density upper level sets $\left\{\mathbf{x} \in \mathbb{R}^{d}: f(\mathbf{x})>\beta\right\}$ are convex for all $\beta \in[0, \infty)$. 
As defined by Kong and Zuo (2010), a distribution $P$ is said to be contiguous if there is no pair of non-intersecting closed halfspaces such that the probability of their union is one.

Denote by $\mathbb{S}^{d-1}$ the unit sphere in $\mathbb{R}^{d}$. Given a distribution $P$ and $\mathbf{x} \in \mathbb{R}^{d}$, the Tukey depth (or halfspace depth) $D$ of $\mathbf{x}$ with respect to $P$ is defined as the minimal probability mass contained in a closed halfspace with $\mathbf{x}$ on its boundary

$$
D(\mathbf{x} ; P)=\inf _{\varphi \in \mathbb{S}^{d-1}} P(H(\mathbf{x}, \varphi)),
$$

where $H(\mathbf{x}, \varphi)=\{\mathbf{x}+\mathbf{y}:\langle\mathbf{y}, \varphi\rangle \geq 0\}$ is a closed halfspace with boundary at $\mathbf{x}$ and inner normal $\varphi$.

Let $p \in\left[0, \sup _{\mathbf{x} \in \mathbb{R}^{d}} D(\mathbf{x} ; P)\right]$. The Tukey depth $p$-region of a distribution $P$ is

$$
D_{p}(P)=\left\{\mathbf{x} \in \mathbb{R}^{d}: D(\mathbf{x} ; P) \geq p\right\}
$$

and the Tukey depth $p$-contour is the topological boundary of the $p$-region.

A result of Kong and Zuo (2010) tells us that if every Tukey depth contour of a contiguous distribution is smooth, then the underlying distribution is completely characterized by its set of depth contours. This means that there does not exist any other contiguous distribution with the same collection of depth contours.

By smoothness of depth contours in $\mathbb{R}^{d}$ we mean that for each $p \geq 0$, the corresponding depth contour can be locally described as a differentiable mapping from $\mathbb{R}^{d-1}$.

The condition of smoothness of depth contours may seem to be weak, but in fact it is quite challenging to verify it for concrete distributions.

The key role in the examples in the following sections will be played by the halfspace function, which can in general be defined as follows.

Definition. Let $P$ be a probability distribution on $\mathbb{R}^{d}$ and $\mathbf{x} \in \mathbb{R}^{d}$. Then the halfspace function of $P$ at $\mathbf{x}$ is the function

$$
\mathcal{G}(\varphi ; \mathbf{x}, P)=P(H(\mathbf{x}, \varphi)) \text { for } \varphi \in \mathbb{S}^{d-1} .
$$

The argument of the distribution $P$ will be omitted when there is no ambiguity. The Tukey depth of $P$ at point $\mathbf{x}$ can be obtained by minimizing $\mathcal{G}(. ; \mathbf{x}, P)$ over its domain.

Now, a key proposition giving a sufficient condition for non-smoothness of a depth contour can be formulated.

Proposition 1. Let $P$ be a contiguous distribution on $\mathbb{R}^{d}, \boldsymbol{x} \in \mathbb{R}^{d}$ such that $D(\boldsymbol{x} ; P)=$ $p>0$, and let $D_{p}(P)$ have non-empty interior. Then the halfspace function attains more than one different global minima at $\boldsymbol{x}$, that is

$$
\liminf _{\varphi \rightarrow \varphi_{1}} \mathcal{G}(\varphi ; \boldsymbol{x})=\liminf _{\varphi \rightarrow \varphi_{2}} \mathcal{G}(\varphi ; \boldsymbol{x})=D(\boldsymbol{x} ; P) \quad \text { for some } \varphi_{1} \neq \varphi_{2},
$$

if and only if the Tukey depth p-contour is not smooth at $\boldsymbol{x}$.

Proof. Assume that (3) holds true. Since (Rousseeuw and Ruts, 1999)

$$
D_{p}(P)=\bigcap\left\{H(\mathbf{y} ; \varphi): \mathbf{y} \in \mathbb{R}^{d}, \varphi \in \mathbb{S}^{d-1}, P(H(\mathbf{y}, \varphi))>1-p\right\},
$$

it can be seen that the two hyperplanes associated with $H\left(\mathbf{x} ; \varphi_{i}\right), i=1,2$ are in fact supporting hyperplanes of the bounded convex set $D_{p}(P)$ at $\mathbf{x}$. This is, however, possible only if $D_{p}(P)$ has a non-differentiable boundary at $\mathbf{x}$ (Giaquinta and Modica, 2012, Theorem 2.36). 
The opposite implication was proved by Massé (2004, Proposition 4.6) for distributions with connected support, but the proof holds for distributions with contiguous support as well.

From now on, we will work in the bivariate $\operatorname{setup}(d=2)$. The domain of $\mathcal{G}$ can then be identified with the interval $[0,2 \pi)$, or even taken to be $\mathbb{R}$, if $\mathcal{G}$ is extended $2 \pi$-periodically.

It will be of use later to transform and simplify (2) at a point $\mathbf{x}=\left(0, y_{0}\right)^{\top}, y_{0}>0$. Assuming $y_{0}$ and the density $f$ are such that

$$
0<\int_{\mathbb{R}} \int_{y_{0}}^{\infty} f(x, y) \mathrm{d} y \mathrm{~d} x<0.5
$$

allows for substitution $\alpha=\tan (\varphi-\pi / 2)$ in $\mathcal{G}$ in $(2)$ to get

$$
\mathcal{H}\left(\alpha ; y_{0}, f\right)=\mathcal{H}\left(\alpha ; y_{0}\right)=\int_{\mathbb{R}} \int_{y_{0}+\alpha x}^{\infty} f(x, y) \mathrm{d} y \mathrm{~d} x=\mathcal{G}\left(\arctan (\alpha)+\frac{\pi}{2} ;\left(\begin{array}{c}
0 \\
y_{0}
\end{array}\right)\right)
$$

Under the assumptions above we can write $D(\mathbf{x} ; P)=\inf _{\alpha \in \mathbb{R}} \mathcal{H}\left(\alpha ; y_{0}\right)$. The following characterization of points of depth regularity will be useful. It is a corollary of Proposition 1.

Proposition 2. Let $P$ be a contiguous distribution on $\mathbb{R}^{2}$ with a density $f$ such that $f(x, y)=f(-x, y)$ for all $x, y \in \mathbb{R}$, and $y_{0}>0$ such that (4) holds true. Then the Tukey depth contour is smooth at $\left(0, y_{0}\right)^{\top}$ if and only if $\mathcal{H}\left(. ; y_{0}\right)$ attains its unique global minimum at zero.

In the sequel, we consider smooth bivariate densities $f$ that can be decomposed into a contour function $\mathfrak{C}: \mathbb{R}^{2} \rightarrow[0, \infty)$ and an integrable radial function $\rho:[0, \infty) \rightarrow[0, \infty)$. The density then takes, up to a multiplicative constant, the form

$$
f(\mathbf{x}) \propto \rho(\mathfrak{C}(\mathbf{x})) \text { for } \mathbf{x} \in \mathbb{R}^{2} .
$$

The contour function is a continuous function whose role is to assess to $\mathbf{x} \in \mathbb{R}^{2}$ a nonnegative number $t$ representing the contour to which $\mathbf{x}$ belongs. For example, $\mathfrak{C}$ could be a norm on $\mathbb{R}^{2}$, or it can be given by the Minkowski functional of a simple closed curve with the origin in its interior. If given by a curve, then the curve represents the contour $\left\{\mathbf{x} \in \mathbb{R}^{2}: \mathfrak{C}(\mathbf{x})=1\right\}$ and all the points $\mathbf{x}$ such that $\mathbf{x} / k$ lies on the curve for $k \geq 0$ are assigned $\mathfrak{C}(\mathbf{x})=k$. The radial function $\rho$ at $t$ evaluates the relative (up to a multiplicative constant) density value for the whole contour of points $\left\{\mathbf{x} \in \mathbb{R}^{2}: \mathfrak{C}(\mathbf{x})=t\right\}$. By choosing $\mathfrak{C}(\mathbf{x})=\|\mathbf{x}\|_{2}^{2}$ (the usual squared $L^{2}$ norm) and $\rho(t)=\exp (-t / 2)$ the standard bivariate Gaussian distribution is obtained.

By Proposition 2 the problem of determining whether $(\mathbf{S})$ is violated at $\left(0, y_{0}\right)^{\top}$ for $P$ can be reduced to checking if the corresponding $\mathcal{H}$ function has a single global minimum at $\alpha=0$. If the density (5) satisfying the assumptions of Proposition 2 is smooth enough, one may simply infer on the second derivative of $\mathcal{H}$ with respect to $\alpha$ at zero

$$
\frac{\partial^{2} \mathcal{H}}{\partial \alpha^{2}}\left(0 ; y_{0}\right)=-2 C \int_{0}^{\infty} x^{2} \rho^{\prime}\left(\mathfrak{C}\left(x, y_{0}\right)\right) \frac{\partial \mathfrak{C}}{\partial y}\left(x, y_{0}\right) \mathrm{d} x
$$

If this is negative for some $y_{0}>0$ such that (4) holds, the Tukey depth contours of $f$ are not smooth at $\left(0, y_{0}\right)^{\top}$. Thus, to establish that a distribution with a smooth density (5), 
symmetric around the $y$-axis, has non-smooth depth contours, it is enough to find $y_{0}>0$ such that (4) holds true and

$$
\int_{0}^{\infty} x^{2} \rho^{\prime}\left(\mathfrak{C}\left(x, y_{0}\right)\right) \frac{\partial \mathfrak{C}}{\partial y}\left(x, y_{0}\right) \mathrm{d} x>0 .
$$

\section{Mixture of Gaussian Distributions}

As an example of a distribution with smooth and strictly unimodal density not satisfying $(\mathbf{S})$, a mixture of bivariate Gaussian distributions will be utilized. Firstly, examine the $\mathcal{G}$ function if the underlying distribution is a bivariate Gaussian with independent components.

Lemma 1. Let $\sigma_{i}^{2}>0, i=1,2, P=N_{2}\left(\left(\begin{array}{l}0 \\ 0\end{array}\right),\left(\begin{array}{cc}\sigma_{1}^{2} & 0 \\ 0 & \sigma_{2}^{2}\end{array}\right)\right), \varphi=\left(\begin{array}{l}\varphi_{1} \\ \varphi_{2}\end{array}\right) \in \mathbb{S}^{1}$ and $\boldsymbol{x} \in \mathbb{R}^{2}$. Then

$$
\mathcal{G}(\varphi ; \boldsymbol{x})=1-\Phi(\langle\boldsymbol{x}, \tilde{\varphi}\rangle),
$$

where $\tilde{\varphi}=\varphi / \sqrt{\left(\sigma_{1} \varphi_{1}\right)^{2}+\left(\sigma_{2} \varphi_{2}\right)^{2}}$ and $\Phi$ is the cumulative distribution function of the standard Gaussian distribution.

Proof. For a random vector $\mathbf{X}$ with distribution $P$, the quantity $\mathcal{G}(\varphi ; \mathbf{x})$ can be expressed as the probability of the event $\{\langle\mathbf{X}, \varphi\rangle \geq\langle\mathbf{x}, \varphi\rangle\}=\{\langle\mathbf{X}, \tilde{\varphi}\rangle \geq\langle\mathbf{X}, \tilde{\varphi}\rangle\}$. In the latter set, $\langle\mathbf{X}, \tilde{\varphi}\rangle$ has a standard Gaussian distribution and the result follows.
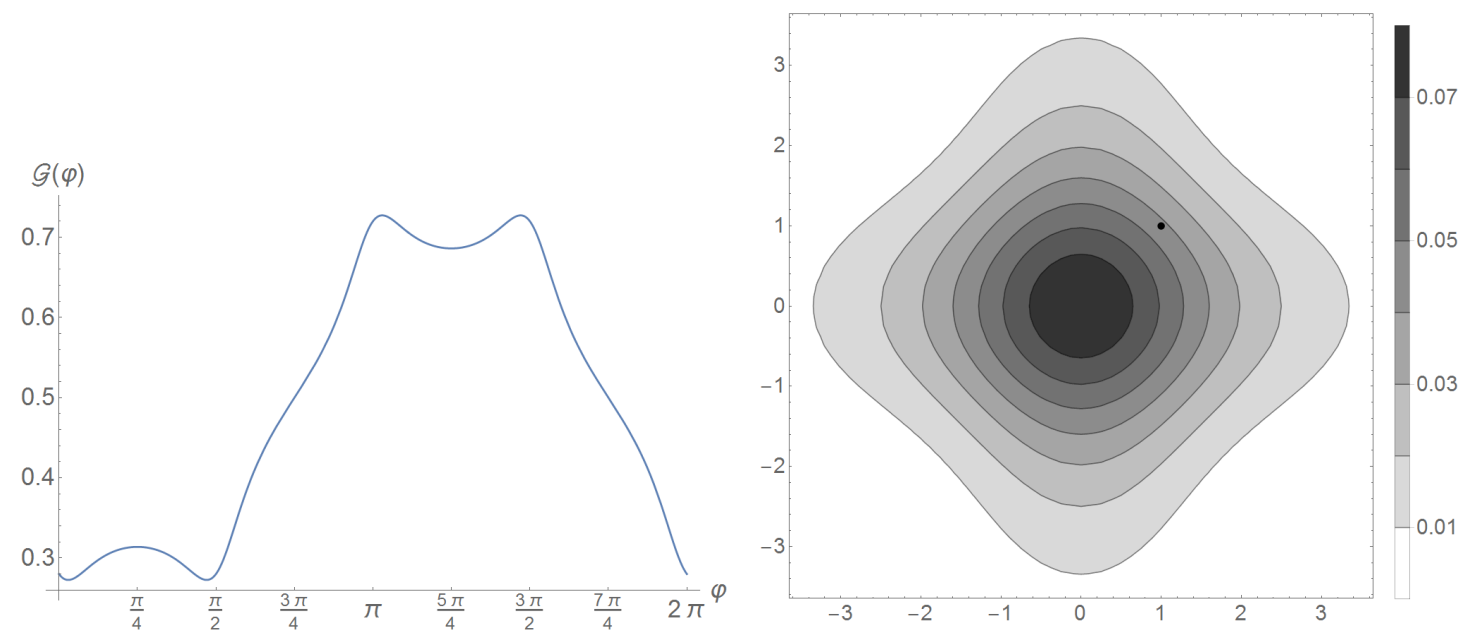

Figure 1. The function $\mathcal{G}(\varphi ; \mathbf{x})$ for $\mathbf{x}=(1,1)^{\top}$, and the density contours of a bivariate Gaussian mixture distribution, $\sigma_{1}=1, \sigma_{2}=4$. The black point on the right hand side is the point of depth irregularity $\mathbf{x}=(1,1)^{\mathrm{T}}$. Two distinct minima of $\mathcal{G}$ are clear to recognize.

Now, let $P$ be a mixture of two bivariate centred Gaussian distributions with covariance matrices $\left(\begin{array}{cc}1 & 0 \\ 0 & 4^{2}\end{array}\right)$ and $\left(\begin{array}{cc}4^{2} & 0 \\ 0 & 1\end{array}\right)$, and equal mixing proportions. Consider $\mathbf{x}=(1,1)^{\top}$. In Fig. 1 we can see the graph of $\mathcal{G}(\varphi ; \mathbf{x}, P)$ and the density contours of $P$. 

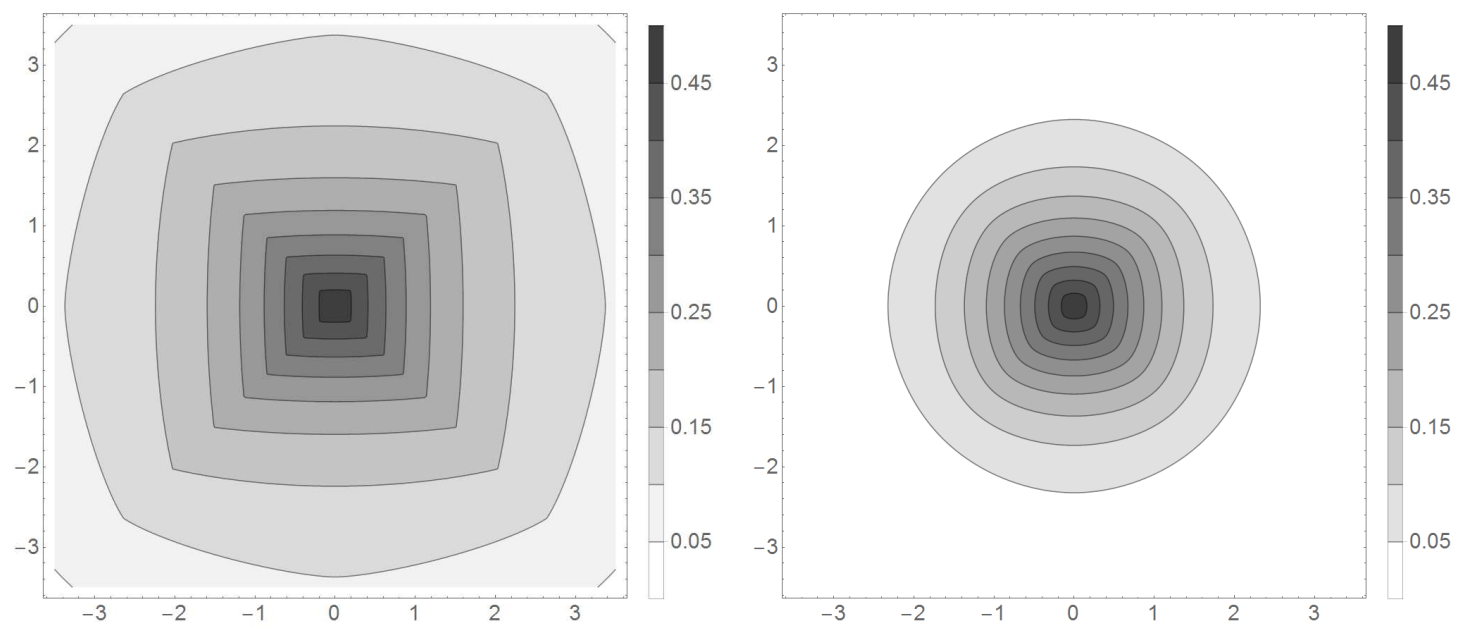

FiguRE 2. Numerically computed Tukey depth contours of two bivariate Gaussian mixture distributions. On the left hand side we see the case $\sigma_{1}=1, \sigma_{2}=4$, where the points of non-smoothness of the form $(x, \pm x)^{\top}$ are evident. On the right hand side $\sigma_{1}=1, \sigma_{2}=\sqrt{3}$. There are no apparent points of non-smoothness of the depth contours.

By Propositions 1 and 2, $P$ does not satisfy $(\mathbf{S})$ if the second partial derivative of $\mathcal{G}$ corresponding to $\mathbf{x}$ at $\varphi=\frac{\pi}{4}$ is negative. This, however, is true, as

$$
\left.\frac{\partial^{2} \mathcal{G}(\varphi ; \mathbf{x}, P)}{\partial \varphi^{2}}\right|_{\varphi=\frac{\pi}{4}}=-\frac{5662 \sqrt{\frac{2}{17 \pi}}}{4913 e^{\frac{2}{17}}}<0,
$$

and $(\mathbf{S})$ is indeed not satisfied for $P$.

In fact, it can be shown that $\frac{\partial^{2} \mathcal{G}(\varphi ; \mathbf{x}, P)}{\partial \varphi^{2}}$ evaluated at $\varphi=\frac{\pi}{4}$ is, for a general choice of variances $\sigma_{1}, \sigma_{2}>0$ in the mixture setup above, negative at every $\mathbf{x}=(x, x)^{\top}$ or $\mathbf{x}=(x,-x)^{\top}$ for $x \in\left(-K\left(\sigma_{1}, \sigma_{2}\right), K\left(\sigma_{1}, \sigma_{2}\right)\right)$, where

$$
K\left(\sigma_{1}, \sigma_{2}\right)=\frac{\sqrt{\sigma_{1}^{6}-3 \sigma_{1}^{4} \sigma_{2}^{2}-3 \sigma_{1}^{2} \sigma_{2}^{4}+\sigma_{2}^{6}}}{\left|\sigma_{1}^{2}-\sigma_{2}^{2}\right| \sqrt{2}} .
$$

For our choice of variances we obtain $K(1,4)=\frac{\sqrt{3281}}{15 \sqrt{2}} \approx 2.700$. Therefore, the "inner" Tukey depth $p$-contours are not smooth at infinitely many different points lying on the axes of the quadrants in $\mathbb{R}^{2}$. The left hand side of Fig. 2 shows a few actual numerically computed Tukey depth contours of the distribution $P$.

Notice, however, that $K\left(\sigma_{1}, \sigma_{2}\right)>0$ only if the ratio of the variances is far enough from one. To be more specific, for $0.268 \approx 2-\sqrt{3}<\sigma_{1}^{2} / \sigma_{2}^{2}<2+\sqrt{3} \approx 3.732$ we have no evidence of violating $(\mathbf{S})$. Actually, numerical computations in such cases suggest (see Fig. 2) that there may not be any points of depth irregularity. This is despite the fact that for all $\sigma_{1}^{2} \neq \sigma_{2}^{2}$ the density of the corresponding Gaussian mixture distribution is neither quasi-concave, nor elliptically symmetrical. 


\section{4. $L^{p}$ Symmetrical Distributions}

Consider now general classes of $L^{p}$ symmetrical distributions in $\mathbb{R}^{2}$ for $1 \leq p \leq \infty$, i.e. distributions with a density $f$ of form (5) with $\mathfrak{C}(\mathbf{x})=\|\mathbf{x}\|_{p}^{p}$ (the $p$ th power of the $L^{p}$ norm) for $\mathbf{x} \in \mathbb{R}^{2}$. For $p=2$ we obtain the class of all absolutely continuous spherically symmetrical distributions.

Note that for $p \in[1, \infty]$ the density upper level sets form boundaries of closed convex sets in $\mathbb{R}^{2}$. Moreover, if $p \in(1, \infty)$, all the level sets of $f$ have smooth boundaries.

If $\rho$ is non-increasing (decreasing) on $[0, \infty)$, we get a distribution with a quasi-concave (strictly quasi-concave) density function. If the following conditions hold

- $\rho$ is smooth on $(0, \infty)$, and

- the right-sided first derivative of $\rho$ vanishes at zero, i.e. $\rho^{\prime}(0+)=0$,

then with a slight abuse of notation we say that $\rho$ is a smooth radial function. This is because under these assumptions the density $f$ is smooth on every line passing through the origin. Provided, in addition, the smoothness of $\mathfrak{C}$ (such as $p \in(1, \infty)$ for $L^{p}$ symmetrical distributions), $f$ itself is a smooth function on the whole space $\mathbb{R}^{2}$.

Dutta et al. (2011) stated that for $p=2$ the Tukey depth contours coincide with the contours of an $L^{2}$ norm, no matter of what form $\rho$ is. Additionally, in Section 2 the authors prove that for any $p \neq 2$ the contours of the density cannot coincide with the Tukey depth contours for $\rho$ decreasing. In the present section, we extend these results by investigating whether the Tukey depth contours of $L^{p}$ symmetrical distributions are smooth or not.

To find an example of a smooth $L^{p}$ symmetrical distribution $P$ such that $(\mathbf{S})$ is violated for $P$, set (for instance) $p=4$ and consider a non-monotonic smooth radial function $\rho(t)=t^{2} \exp (-t)$. The integral in formula (7), providing a sufficient condition for nonsmoothness of depth contours at $y_{0}>0$, can be written as

$$
\int_{0}^{\infty} x^{2} \rho^{\prime}\left(\mathfrak{C}\left(x, y_{0}\right)\right) \frac{\partial \mathfrak{C}}{\partial y}\left(x, y_{0}\right) \mathrm{d} x=-\frac{1}{16} \exp \left(-y_{0}^{4}\right) y_{0}^{3}\left(16 y_{0}^{8}-8 y_{0}^{4}-3\right) \Gamma\left(\frac{3}{4}\right)
$$

where $\Gamma$ is the Euler gamma function. But this is positive indeed, for all $y_{0} \in\left(0, \frac{\sqrt[4]{3}}{\sqrt{2}}\right) \approx$ $(0,0.93)$, and $(\mathbf{S})$ is violated for $P$. The left panel of Fig. 3 presents the density contours for this $L^{4}$ symmetrical distribution, with indication of the point of depth irregularity $(0,0.5)^{\top}$.

Similar examples for other values $p \neq 2$ are immediately available by choosing a proper smooth radial function $\rho$ increasing quickly enough in the right neighbourhood of 0 . In conclusion, the following proposition holds.

Proposition 3. For any $p \in[1, \infty], p \neq 2$, there exists an $L^{p}$ symmetrical distribution $P$ with smooth density such that $(\mathbf{S})$ is not satisfied for $P$.

\section{Symmetrical Quasi-Concave Distributions}

In Sections 3 and 4 we considered various unimodal distributions with smooth densities and concluded that these properties are not enough for condition $(\mathbf{S})$ to be satisfied. This leads to a natural question; if a distribution $P$ with a smooth density is (strictly) quasiconcave, is then necessarily $(\mathbf{S})$ true for $P$ ? In other words, as the Tukey depth is always quasi-concave; is the smoothness preserved for Tukey depth contours, provided the density is also quasi-concave? 

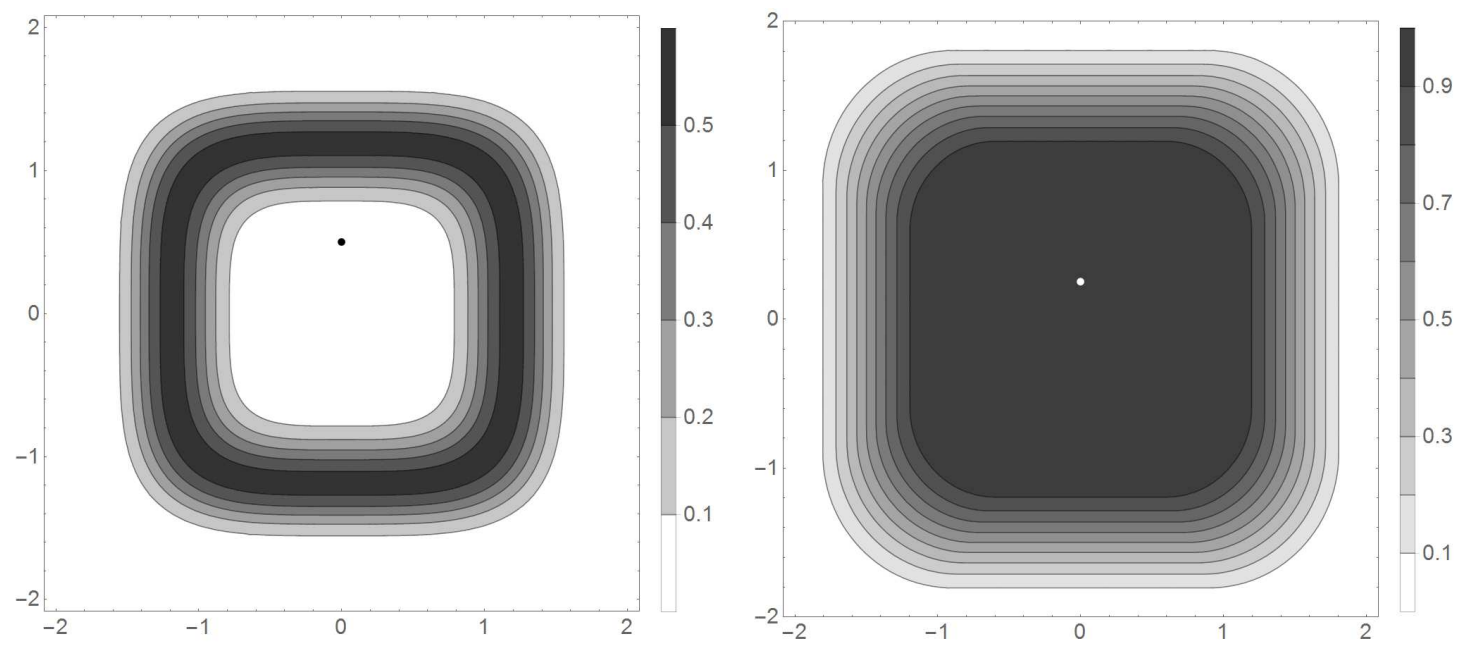

Figure 3. The (unscaled) density contours of the $L^{4}$ symmetrical (left) and the centrally symmetrical (right) distribution along with their points of depth irregularity $\mathbf{x}=(0,0.5)^{\top}$ (black point) and $\mathbf{x}=(0,0.25)^{\top}$ (white point), respectively. At these points, the corresponding Tukey depth contours are not smooth.

The answer to this question is, however, negative. If we leave the setup of $L^{p}$ symmetrical distributions, it is possible to construct a density of this type with infinitely many points of depth irregularity. To see this, consider the following two examples.

5.1. Centrally Symmetrical Distribution. By Rousseeuw and Ruts (1999, Section 5.4 ), a uniform distribution on a unit square in $\mathbb{R}^{2}$ does not satisfy $(\mathbf{S})$. Here, we extend this example to obtain a centrally symmetrical distribution $P$ with a smooth quasiconcave density such that $(\mathbf{S})$ is still violated for $P$.

Define a smoothed square boundary on the set $\left\{(x, y)^{\top} \in \mathbb{R}^{2}: 0<x<y\right\}$ as a function

$$
S(x)= \begin{cases}1 & \text { if } 0 \leq x \leq 0.5 \\ -\frac{1}{2}+\sqrt{2-2 x}+x & \text { if } 0.5<x<\frac{7}{8}\end{cases}
$$

and extend it symmetrically first around the $x=y$ axis, then around $y=0$, and finally around $x=0$. What we get is a simple closed curve $\gamma$ delimiting a square with smoothed vertices. Inflating it using Minkowski functional we obtain the contour function

$$
\mathfrak{C}(x, y)=\left\{t \in[0, \infty): \frac{1}{t}(x, y)^{\top} \in \gamma\right\}
$$

Take the smooth non-increasing radial curve

$$
\rho(t)= \begin{cases}1 & \text { for } t \in[0,1] \\ 2(t-1)^{3}-3(t-1)^{2}+1 & \text { for } t \in(1,2) \\ 0 & \text { for } t \geq 2\end{cases}
$$

Now it is enough to realize that the function $\mathcal{H}$ at point $0<y_{0}<0.5$ is constant in an open interval $\left(-0.5+y_{0}, 0.5-y_{0}\right)$ around $\alpha=0$. Thus, by Proposition $1,(\mathbf{S})$ is not true for $P$. To see this, consider the density values on the set difference between the 
hyperplanes $\left\{x>y_{0}\right\}$ and $\left\{x>y_{0}+\alpha x\right\}$. Owing to the constancy of $\rho$ around the origin and the fact that for the contour function it holds that

$$
\mathfrak{C}(x, y)=|x| \text { for }|y|<\left|\frac{x}{2}\right|,
$$

it can be seen that for some constant $C>0$

$$
f\left(x, y_{0}+\alpha x\right)=C \rho\left(\mathfrak{C}\left(x, y_{0}+\alpha x\right)\right)=C \rho(|x|)=f\left(-x, y_{0}-\alpha x\right) \text { for }|\alpha|<0.5-y_{0} .
$$

Consequently, $\mathcal{H}\left(0 ; y_{0}\right)=\mathcal{H}\left(\alpha ; y_{0}\right)$ for all $|\alpha|<0.5-y_{0}$. See Fig. 3 (right panel) for the density contours of this centrally symmetrical distribution, with indication of the point of depth irregularity $\mathbf{x}=(0,0.25)^{\mathrm{T}}$. In conclusion, the following (negative) result holds.

Proposition 4. There exists a centrally symmetrical distribution $P$ with smooth quasiconcave density such that $(\mathbf{S})$ is not satisfied for $P$.

5.2. Strictly Quasi-Concave Distribution. In the previous example, in the expression (6) for the second derivative of $\mathcal{H}$ we have by (8) that $\frac{\partial^{2} \mathcal{H}}{\partial \alpha^{2}}\left(0 ; y_{0}\right)=0$. Thus, to construct a distribution with a smooth density (5) and a decreasing smooth $\rho$, we see by (7) that it is necessary that

$$
\frac{\partial \mathfrak{C}}{\partial y}\left(x, y_{0}\right)<0
$$

is true for as many $x \in \mathbb{R}$ as possible, for the Tukey depth contour to be non-smooth at $\left(0, y_{0}\right)^{\top}$.

Combining this remark with the fact that the set $\left\{\mathbf{x} \in \mathbb{R}^{2}: \mathfrak{C}(\mathbf{x})<1\right\}$ should be convex, we can construct a strictly quasi-convex smooth density of the form (5) such that (7) holds. However, we must abandon the class of centrally symmetrical distributions since in that case the strict quasi-concavity rules out condition (9).

Consider an equilateral triangle with vertices $(0,-4)^{\top},(\sqrt{2}(-1+\sqrt{3}), \sqrt{2}(1+\sqrt{3}))^{\top}$ and $(\sqrt{2}(1-\sqrt{3}), \sqrt{2}(1+\sqrt{3}))^{\top}$. As pointed out by Rousseeuw and Ruts (1999), the uniform distribution on such a triangle also violates $(\mathbf{S})$. Smoothing of the vertices of the triangle will be done again by second order Bézier curves; for the bottom vertex this curve can be described explicitly by

$$
S(x)= \begin{cases}x^{2}-\frac{13}{4} & \text { for }|x|<\frac{\sqrt{3}}{2} \\ -4+\sqrt{3}|x| & \text { for } \frac{\sqrt{3}}{2} \leq|x| \leq \sqrt{3} .\end{cases}
$$

The smoothing curves for the remaining two vertices are obtained by rotating the curve $y=S(x)$ by the angles $2 \pi / 3$ and $4 \pi / 3$ in polar coordinates. It is easy to see that (9) holds true for some $x$ for any $y_{0}>0$.

Plugging in the smooth, strictly decreasing radial function $\rho(t)=\exp \left(-t^{2}\right)$ and performing the same computation as in Section 5.1 for $y_{0}=0.5$, we finalize the example. Numerical results can be found in Fig. 4. From the graph of $\mathcal{H}(\alpha ; 0.5)$ in the neighbourhood of $\alpha=0$ it is clear that at zero it attains its local maximum, and thus by Proposition 2 the Tukey depth contours are not smooth at $(0,0.5)^{\top}$. We omit the analytical computations of the integrals in (7) for brevity. This example leads to the following statement.

Proposition 5. There exists a distribution $P$ with smooth strictly quasi-concave density such that $(\mathbf{S})$ is not satisfied for $P$. 

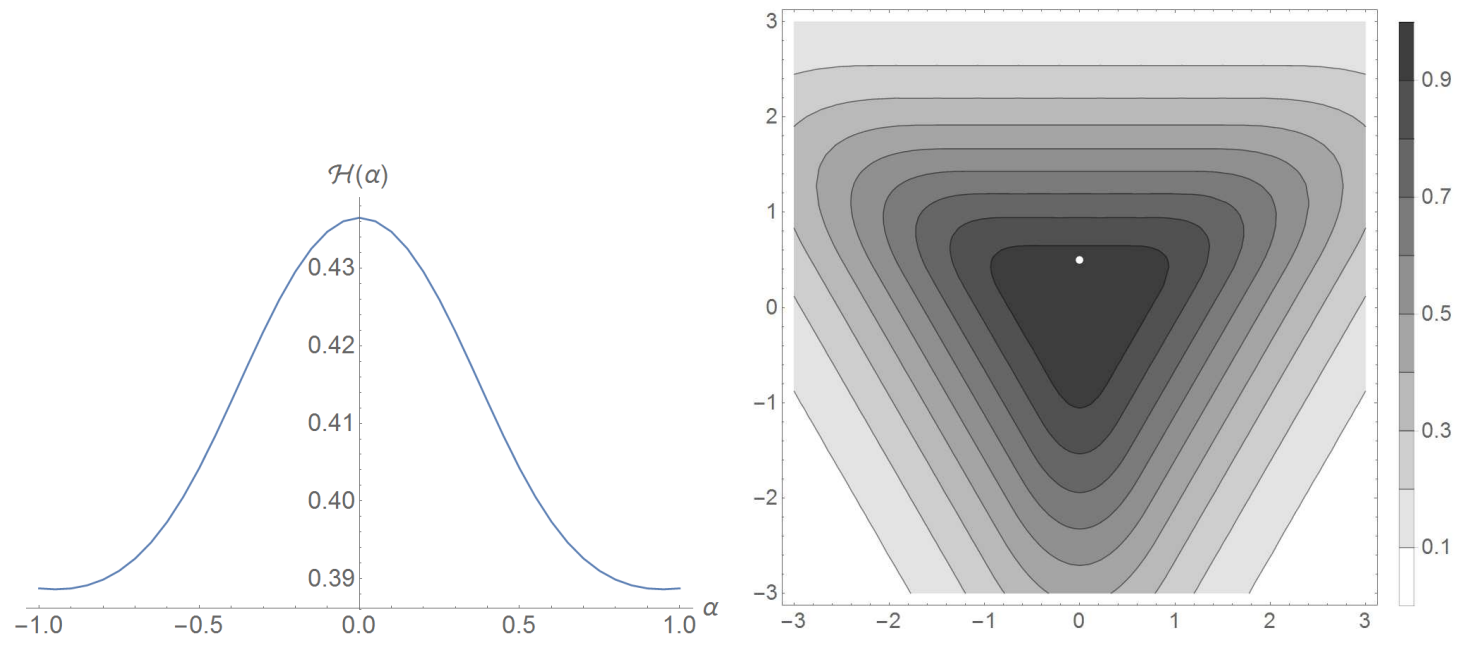

FiguRE 4. The function $\mathcal{H}(\alpha ; 0.5)$ (left) in the neighbourhood of $\alpha=0$ and the (unscaled) density contours of a strictly quasi-concave distribution (right) along with the point of depth irregularity $\mathbf{x}=(0,0.5)^{\top}$ (white point). At $\alpha=0$ the function has a local maximum, thus the corresponding Tukey depth contours are not smooth at $\mathbf{x}$.

\section{Conclusion}

In this paper we showed that smoothness of the Tukey depth contours can be violated even for very well-behaved multivariate probability distributions. Consequently, the distribution-by-depth characterization result of Kong and Zuo (2010) is in that sense of limited use, and sure to hold only for the case of elliptically symmetrical distributions. Similarly, the asymptotic behaviour of the Tukey depth process, and the Tukey depth regions (Massé and Theodorescu, 1994) appears to be rather complex already for very simple distributions. In particular, at non-smooth points the limiting distributions of the depth processes may be non-Gaussian, or the processes may even fail to converge weakly (Massé, 2004, Theorem 2.4). Moreover, subtle changes in the multivariate probability distribution may have great impact on the smoothness of the Tukey depth contours, as was seen in Section 3. Therefore we may mention the Tukey depth as non-robust in this sense. This finding complements the study of Romanazzi (2001) who showed that the influence function of the empirical Tukey depth is bounded.

Acknowledgement. The authors are grateful to two referees for their helpful suggestions on an earlier version of the manuscript. This research is supported by the IAP research network nr. P7/06 of the Federal Science Policy (Belgium). The first author gratefully acknowledges support from the GOA/12/014-project of the Research Fund KU Leuven. The work of the second author was partially supported by the Czech Science Foundation project No. P402/12/G097 "DYME — Dynamic Models in Economics". Currently he is a Research Assistant of the Research Foundation - Flanders, and acknowledges support from this foundation.

\section{REFERENCES}

Cuesta-Albertos, J. A. and Nieto-Reyes, A. (2008). The Tukey and the random Tukey depths characterize discrete distributions. J. Multivar. Anal., 99:2304-2311. 
Donoho, D. L. and Gasko, M. (1992). Breakdown properties of location estimates based on halfspace depth and projected outlyingness. Ann. Statist., 20(4):1803-1827.

Dutta, S., Ghosh, A. K., and Chaudhuri, P. (2011). Some intriguing properties of Tukey's half-space depth. Bernoulli, 17(4):1420-1434.

Giaquinta, M. and Modica, G. (2012). Mathematical analysis. Foundations and advanced techniques for functions of several variables. Boston, MA: Birkhäuser.

Hassairi, A. and Regaieg, O. (2008). On the Tukey depth of a continuous probability distribution. Statist. Probab. Lett., 78(15):2308-2313.

Kong, L. and Zuo, Y. (2010). Smooth depth contours characterize the underlying distribution. Journal of Multivariate Analysis, 101(9):2222-2226.

Koshevoy, G. A. (2002). The Tukey depth characterizes the atomic measure. J. Multivariate Anal., 83(2):360-364.

Massé, J.-C. (2004). Asymptotics for the Tukey depth process, with an application to a multivariate trimmed mean. Bernoulli, 10(3):397-419.

Massé, J.-C. and Theodorescu, R. (1994). Halfplane trimming for bivariate distributions. J. Multivariate Anal., 48(2):188-202.

Nolan, D. (1992). Asymptotics for multivariate trimming. Stochastic Process. Appl., 42(1):157-169.

Romanazzi, M. (2001). Influence function of halfspace depth. J. Multivariate Anal., $77(1): 138-161$.

Romanazzi, M. (2002). Corrigendum: "Influence function of halfspace depth" [J. Multivariate Anal. 77 (2001), no. 1, 138-161; MR1838717 (2002h:62158)]. J. Multivariate Anal., 81(1):187-188.

Rousseeuw, P. J. and Ruts, I. (1999). The depth function of a population distribution. Metrika, 49(3):213-244.

Struyf, A. and Rousseeuw, P. J. (1999). Halfspace depth and regression depth characterize the empirical distribution. J. Multivariate Anal., 69(1):135-153.

Tukey, J. W. (1975). Mathematics and the picturing of data. Proc. int. Congr. Math., Vancouver 1974, Vol. 2, 523-531 (1975).

Zuo, Y. and Serfling, R. (2000). General notions of statistical depth function. Ann. Stat., 28(2):461-482. 